\title{
Spoken Language Identification Using Deep Learning
}

\author{
Gundeep Singh $\mathbb{D},{ }^{1}$ Sahil Sharma $\mathbb{D},{ }^{1}$ Vijay Kumar $\mathbb{D},{ }^{2}$ Manjit Kaur $\mathbb{D},{ }^{3}$ Mohammed Baz $\mathbb{D},{ }^{4}$ \\ and Mehedi Masud $\mathbb{1}^{5}$ \\ ${ }^{1}$ Computer Science and Engineering Department, Thapar Institute of Engineering and Technology, Patiala, India \\ ${ }^{2}$ Computer Science and Engineering Department, National Institute of Technology, Hamirpur, India \\ ${ }^{3}$ School of Engineering and Applied Sciences, Bennett University, Greater Noida, India \\ ${ }^{4}$ Department of Computer Engineering, College of Computer and Information Technology, Taif University, P.O. Box. 11099, \\ Taif 21994, Saudi Arabia \\ ${ }^{5}$ Department of Computer Science, College of Computer and Information Technology, Taif University, P.O. Box. 11099, \\ Taif 21994, Saudi Arabia
}

Correspondence should be addressed to Mehedi Masud; mmasud@tu.edu.sa

Received 6 July 2021; Accepted 3 September 2021; Published 21 September 2021

Academic Editor: Ciro Castiello

Copyright $\odot 2021$ Gundeep Singh et al. This is an open access article distributed under the Creative Commons Attribution License, which permits unrestricted use, distribution, and reproduction in any medium, provided the original work is properly cited.

The process of detecting language from an audio clip by an unknown speaker, regardless of gender, manner of speaking, and distinct age speaker, is defined as spoken language identification (SLID). The considerable task is to recognize the features that can distinguish between languages clearly and efficiently. The model uses audio files and converts those files into spectrogram images. It applies the convolutional neural network $(\mathrm{CNN})$ to bring out main attributes or features to detect output easily. The main objective is to detect languages out of English, French, Spanish, and German, Estonian, Tamil, Mandarin, Turkish, Chinese, Arabic, Hindi, Indonesian, Portuguese, Japanese, Latin, Dutch, Portuguese, Pushto, Romanian, Korean, Russian, Swedish, Tamil, Thai, and Urdu. An experiment was conducted on different audio files using the Kaggle dataset named spoken language identification. These audio files are comprised of utterances, each of them spanning over a fixed duration of 10 seconds. The whole dataset is split into training and test sets. Preparatory results give an overall accuracy of $98 \%$. Extensive and accurate testing show an overall accuracy of $88 \%$.

\section{Introduction}

Spoken language identification (SLID) is recognizing the language being a talk by an anonymous speaker from an audio clip. Humans are the most error-free language identification system [1]. There are various implementations of spoken language identification like creating front ends for multilanguage speech identification systems, automatic customer routing in call centers, monitoring, and web information retrieval [2]. The SLID system has three main parts, data collection, feature removal, and language classification, as shown in Figure 1. An essential for developing and evaluating a speech recognition system is the accessibility of a suitable database [3].

Different methods have been proposed to figure out the problems of automatic language identification with the acoustic phonetics approach [1,4]. Encouragement in the deep learning field has empowered researchers to use GANs for language identification for robustness on unsupervised and semisupervised tasks [5]. Support vector machines (SVM) classifiers do not work well on short utterances, giving less accuracy [6]. Conventional identification systems are supported on i-vector systems for spoken language processing tasks, which are inefficient [7].

To resolve the problems which are mentioned above, a log-Mel spectrum is used to generate the spectrograms of audio snippets, which can record or store the frequency of particular audio utterances. It is efficient and fast, and further, we can apply the convolutional neural network (CNN) technique to classify different languages. This work has been done using the spectrum technique using deep learning by the authors [8]. Many researchers are working 


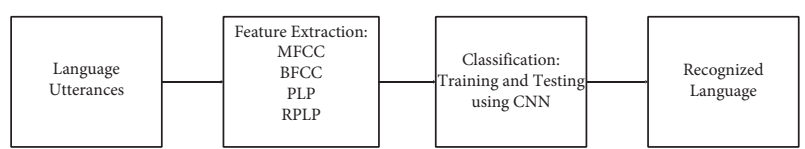

FIGURE 1: Schematic diagram of the spoken language identification system.

on image creations and image identification using a deep learning technique that gives good results and better accuracy in $2 \mathrm{D}[9,10], 2.5 \mathrm{D}[11]$, and $3 \mathrm{D}[12,13]$ domain. There is a challenge to identify spoken language with different genders, other age groups, and various accents. There is noise in the background in some of the audio clips, so it is very hard to identify the language. A deep learning $\mathrm{CNN}$ technique is proposed to draw out the attributes. Figure 2 shows the phases of the proposed spoken language identification framework. A prediction is made by a model, which can easily identify classification in the proposed framework.

1 A novel deep learning-based model is proposed to apply the convolutional neural network (CNN) to draw out attributes from images.

2 The proposed model is analyzed with different deep learning and machine learning techniques over four datasets.

3 The proposed approach differs from other state-of-theart methods on various evaluation metrics and shows the comparison with different techniques.

The formation of work is as follows: Section 2 represents an explorative concept of spoken language identification using CNN. Section 3 discusses past studies in the language identification field. Section 4 discusses the model architecture of raw waveforms and log-Mel spectrogram images. Section 5 represents an experimental result. In Section 6, consequences and results are discussed. Finally, Section 7 concludes the paper.

\section{Background}

This section discusses the preliminary concepts of spoken language identification using $\mathrm{CNN}$, spectrograms, and Multinomial Naïve Bayes.

2.1. Spoken Language Identification Using CNN. The process of spoken language identification using the $\mathrm{CNN}$ technique uses spectrograms of raw audio signals as input to a convolutional neural network (CNN) $[8,14]$. A spoken language identification dataset is collected and preprocessed for the training phase. The main focus is on preprocessing, in which we convert audio utterances in spectrogram images. After that, data is portioned into the training and testing phase. Furthermore, we apply CNN to extract features from it. After the training is completed, the test dataset is used for validation. The prediction accuracy is calculated based on the model's performance in the validation phase.

2.2. Generation of Spectrogram. The spectrogram refers to representing the frequencies on the image that are present

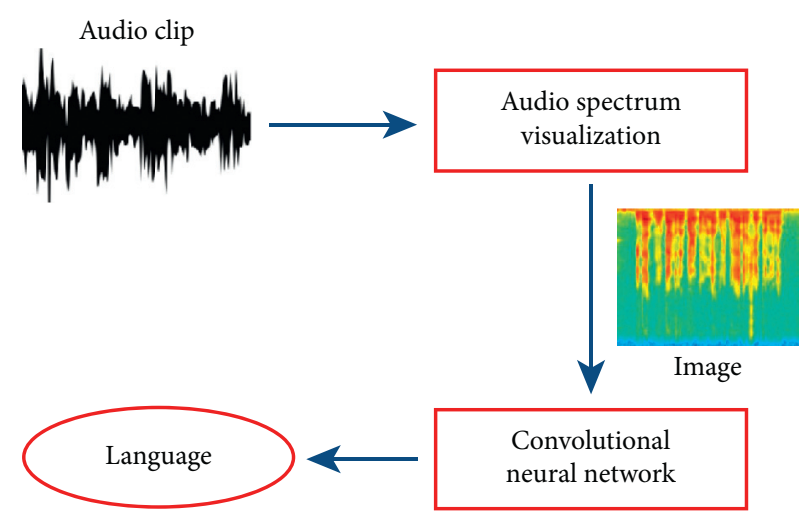

FIgUre 2: Phases of spoken language identification using spectrograms.

on a signal over time. The signal's frequency gives rise from a time series signal of data points using Fast Fourier Transform (FFT). Fast Fourier Transform (FFT) can be put into time-series data to calculate the magnitude of the frequency for a fixed moment in time. The process of time-series data is first windowed, usually in small chunks, and the FFT data is kept together to form the spectrogram images, which empowers us to see how fast the frequencies improve.

Since the work was done to generate spectrograms on audio clips or utterances of data, then data was regenerated into Mel spectrograms, known as spectrograms images. The conversion frequencies from $f$ hertz type to $m$ mels are shown in Figures 3 and 4:

$$
m=2595 \log _{10}\left(1+\frac{f}{700}\right) .
$$

2.3. Bernoulli Naïve Bayes. Bernoulli Naïve Bayes uses discrete data and it works on Bernoulli distribution. The main feature of Bernoulli Naïve Bayes is that it accepts features only as binary values like true or false, yes or no, 0 and 1 , success or failure, and so on. As they deal with binary values, let us consider ' $p$ ' as the probability of success and ' $q$ ' as the probability of failure and $q=1-p$, for a random variable ' $X$ ' in Bernoulli distribution:

$$
p(x)=P[X=x]= \begin{cases}q=1-p, & x=0, \\ p, & x=1,\end{cases}
$$

where ' $x$ ' is in binary form like 0 or 1 . The Bernoulli Naïve Bayes machine learning classifier is based on

$$
p\left(x_{i} \mid y\right)=P(i \mid y) x_{i}+(1-P(i \mid y))\left(1-x_{i}\right) .
$$

\section{Related Work}

Literature [15] proposed a deep learning-based spoken language identification system. It combines DNNs and $i$ vectors to increase efficiency. Every hidden layer includes 2560 neurons with a ReLU function. They use the softmax activation function in the last layer. It compares a DNN and $i$-vectors on two different datasets: $\mathrm{LRE}^{\prime} 09$ and Google 5M 


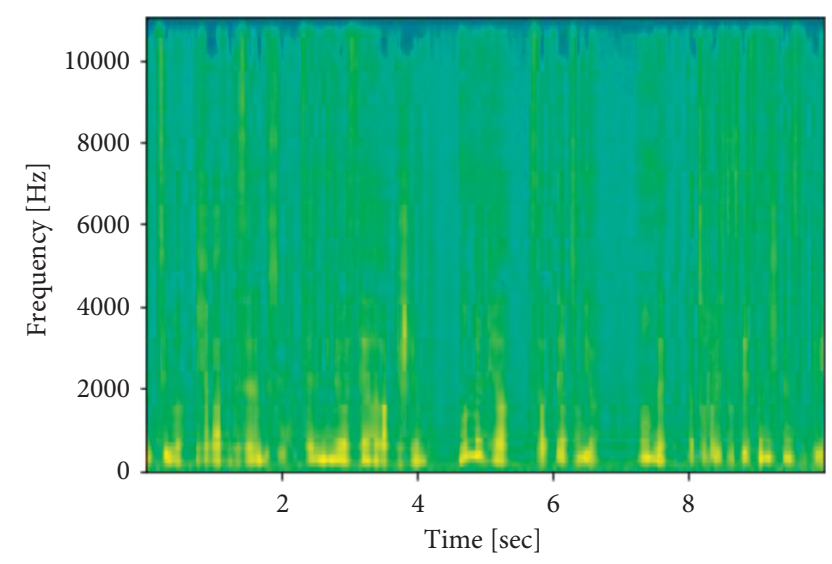

Figure 3: Generated spectrogram from an English audio file.

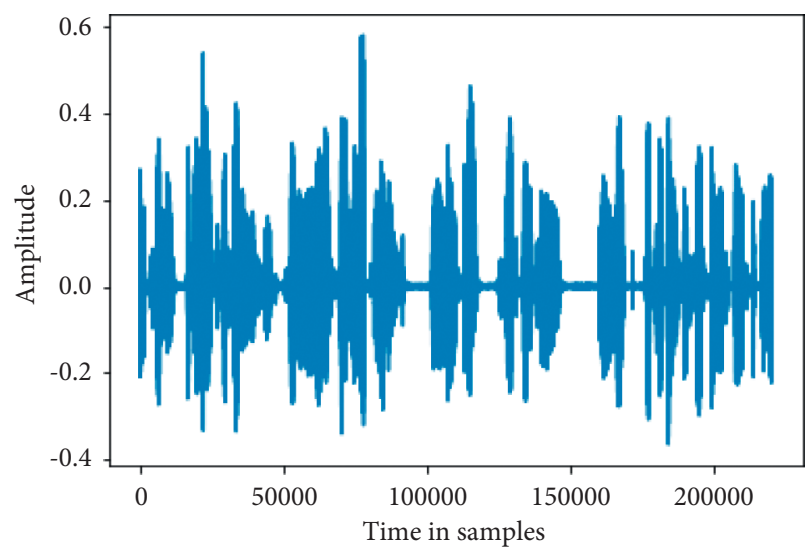

Figure 4: Wavelength of audio.

lid corpus. With these two approaches, they failed to achieve good results. They also compare the performance with Equal Error Rate (EER), where they got an average of $9.58 \%$ using the DNN model. Literature [16] proposed a method that can improve generalization to identify short speech-language using triplet entropy loss with the help of CNN; they combine cross-entropy loss (CEL) and triplet loss through which they generalize the data and use it on Slavic languages. They use ResNet50 pretrained model and use the softmax function at the last layer. It uses an Adam optimizer with the learning rate (LR) of $10 \mathrm{e}-4$, uses the batch size of 32 , and ridge-based regularization to reduce overfitting. The top accuracy they achieved was $78 \%$ using triplet loss. They showed Triplet Entropy loss is better than cross-entropy loss, but they failed to achieve good performance.

Literature [17] proposed an unsupervised neural-based model which can be used in spoken language identification and can decrease the distribution variance on both attributes and classifiers for the training and testing datasets. It proposed the optimal transport (OT) method to measure the distribution of the discrepancy. The Time Delay Neural Network (TDNN) framework is used for determining training and test set adaption. Literature [18] proposed a deep neural network-based model which identifies a Slavic language or those languages which are similar. They created the model with two parameters: segment level feature extractor and language classifier. The model uses the $\mathrm{CNN}$ with 128,256 , and 512 filters with 5, 10, and 10 for each layer with stride 1 at each layer. They use two techniques, Baseline LID and Robust LID. In baseline LID, they got 53.25 as average accuracy, and in robust LID, they got 87.32 as average accuracy. Literature [19] proposed a framework that combines CNN and LSTM system that uses CTC loss function to train the model. In this, audio clips are converted into spectrograms, further CNN is applied to extract the features from it, and further LSTM is used to store the data from previous layers. The speech signal is sampled at $16 \mathrm{KHz}$ with window size $200 \mathrm{~ms}$, and window stride with $100 \mathrm{~ms}$. This method gives an accuracy between $74 \%$ to $76 \%$.

Literature [20] proposed a capsule network framework for identifying spoken language identification systems. In CapsNet, a convolutional layer has a total of 128 kernels with the size of $(9,9,1)$ and a step size of 1 with ReLU function. It divided the CapsNet into two parts: encoder and decoder. The first 4 layers represent the encoder, and the last 3 layers represent the decoder. They achieved an accuracy of $91.80 \%$ with 5-second audio clips using the CapsNet approach. Literature [21] proposed various feature selection methods like top- $k$ selection, forward feature selection, and recursive feature elimination, so the model can work efficiently. In the first phase, preprocessing is done in which it removes the punctuations, emoticons, links, hashtags, URLs, then removes the less important words using the English stop words, and then removes redundant data from the dataset. The top-k feature method performs well; it selects 550 features compared to the other methods. Literature [22] proposed a Recurrent Neural Network Transducer (RNN-T) for speech recognition and spoken language identification. They use two languages pair: English-Spanish and EnglishHindi. The RNN-T framework uses 5 encoder LSTM layers with 1024 units and 2 decoder LSTM layers with 1024 units; a 512-d embedding layer was used as a decoder input. The discrete fracture network (DFN) has 512 hidden neurons, along with tanh and softmax functions.

Literature [23] proposed a technique that associates with acoustic level representations with embedding on Automatic Speech Recognition, which gives a 50\% reduction in error rate. They used 64-d log-Mel feature extractors for training on a $25 \mathrm{~ms}$ window with a $10 \mathrm{~ms}$ overlap. The first 3 LSTM layers comprise 768 units, and all the data is further passed to FC layers and softmax function. At last, they used a semisupervised technique to increase the accuracy and good results of the model. Literature [24] proposed a signal combination approach for language identification. They used a deep learning model to combine the signals from recognizers with the baseline, which uses low-level acoustic signals. It helps to decreases the error rate from $5.50 \%$ to $4.30 \%$. They work with 11 different models and use ReLU, dropouts, Adam, batch normalization, and various other attributes to get good results. Literature [25] proposed a system with an acoustic model and a context-aware model. They created the model with 4 convolutional layers with 128 units, 4 fully connected layers with the 1024 units, 1 fully connected layer with the 512 units, 1 temporal pooling layer 
with a mean and standard deviation, 1 fully connected layer with 1024 units, and at last softmax function is used with unit 1. With this framework, they got $97.0 \%$ accuracy. Literature [5] proposed a conditional GANs classifier framework for language identification, choosing GANs is a better option on large datasets, giving good results. $2 \times 2$ is used to perforate upsampling, $5 \times 5$ Conv. 1 , tanh, and an output (49) tanh is used. With this framework, they achieved 97\% accuracy. Table 1 summarizes the previous studies, features, and results as discussed above.

\section{Proposed Spoken Language Identification Framework}

This section discusses the motivation and the spoken language identification framework.

4.1. Motivation. Various state-of-the-art results on various audio classification tasks have been obtained by using logMel spectrograms of raw audio, like features, which convert the audio utterance into images [8]. CNN gives an excellent performance gain in classification on these features [14]. The motivation of work has come from these studies. The computation time is more for converting audio into spectral images, giving us a new direction to develop the computationally efficient and more accurate spoken identification technique.

4.2. Proposed Spoken Identification Framework. The proposed deep learning-based spoken language identification framework: while designing this framework, audio utterances are converted into spectrograms based on their frequency and time. After this, a convolutional neural network (CNN) is applied to images to extract their features for classification. At last, the softmax activation function is applied for multilanguag classification.

4.3. Preprocessing. In the preprocessing phase, data augmentation is used to solve class imbalance problems. Data augmentation reduces overfitting and acts as a regularizer when training a model. With the help of data augmentation, it can increase the amount of data by adding some modifications of existing data like crop, rotate, flip, shearing, and much more effects. The use of data augmentation is good while using transfer learning models works well on more data and predicts good results.

4.4. Description of Features. The duration of each audio is 10 seconds (sharp) with a sample rate of 22050, a bit depth of 16 bits, and channels 1, and each audio file is a Free Lossless Audio Codec (FLAC) audio sample. The dataset is divided into two directories: train, which contains (73080) samples, and test, which contains (540) samples, with three languages English, German, and Spanish. Several audio transformations are applied, like pitch, speed, and noise. It contains the voice of 90 original speakers of male and female.
4.5. Model Description. In the model description, it describes the framework to all the models for the experiment purpose:

a) An appropriate pooling layer always follows every convolutional layer. It helps to include the explosion of attributes and keeps the model small and efficient.

b) Each convolution layer is followed by the dropout layer, ReLU, and batch normalization. The batch normalization is responsible for the convergence of learned representations.

c) Finally, a dense layer is used, which acts as an output layer of the model.

4.6. Model Characteristic: $2 D$ ConvNet. Table 2 presents a specific layer-by-layer explanation of the model with its different hyperparameters.

4.7. Model Characteristic: Word Embedding. Table 3 presents a layer-by-layer explanation of the model and the details of hyperparameters.

The above word embedding model is a pretrained model by Keras use for experiments for language identification. The first step is to download the stop words using the nltk library; after that, there are 22 languages in the dataset like Arabic, Chinese, Dutch, English, Estonian, French, Hindi, Indonesian, Japanese, Romanian, Korean, Latin, Persian, Portuguese, Pushto, Russian, Turkish, Swedish, Tamil, Thai, Urdu, and Spanish. Each language contains 1000 rows in the dataset. After completing the preprocessing, then further divide the data into the train and test set. Then, apply the word embedding model to the dataset at the output layer; softmax is used because there are 22 classes. With this model, the achieved accuracy is $95 \%$.

4.8. Model Details: Bernoulli Nä̈ve Bayes. This approach uses the Bernoulli Naïve Bayes machine learning technique to identify language from a given dataset. In a preprocessing step, all the data are first split into $X$ and $Y$ and then encode the data using a label encoder library. Following that, perform data cleaning to convert all the sentences into lower case. Then, the Naïve Bayes approach is applied, which takes 29.7 seconds to fit in the model and gives me $93.0 \%$ accuracy. Table 4 shows the metrics that are performed using Naïve Bayes.

\section{Experimental Results and Discussion}

This section contains results and a discussion of different techniques. All the details regarding the use of datasets, hyperparameter settings, evaluation metrics, and computational time analysis of the different proposed approaches are illustrated.

5.1. Datasets. The experiment of the different techniques is implemented using the four datasets, spoken language identification [30], language identification dataset [31], 
TABle 1: Review of previous studies along with results.

\begin{tabular}{|c|c|c|c|c|c|c|}
\hline Year & Model basis & Features & Languages & Acc. & Remarks & Ref. \\
\hline 2021 & $\begin{array}{l}\text { PLDA logistic } \\
\text { regression }\end{array}$ & $\begin{array}{l}i \text {-vector } x \text { - } \\
\text { vector }\end{array}$ & Javanese, Sundanese, Minang & $96 \%$ & $\begin{array}{c}\text { PLDA and logistic classifiers are used } \\
\text { with } x \text {-vector and } i \text {-vector feature } \\
\text { extraction. }\end{array}$ & {$[26]$} \\
\hline 2021 & $\begin{array}{l}\text { CNN ResNet50 } \\
\text { RNN }\end{array}$ & MFCC & $\begin{array}{l}\text { Iba, Kab, Sun, Ind, Eus, Jav, Tam, } \\
\text { Tel, Kan, Hin, Tha, Rus, Cnh, Eng, } \\
\text { Por, Mar }\end{array}$ & $53 \%$ & $\begin{array}{l}\text { Submit three different systems named } \\
\text { Lipsia, Anlirika, and NTR with different } \\
\text { specifications. }\end{array}$ & {$[27]$} \\
\hline 2021 & $\begin{array}{l}\text { Self-attentive } \\
\text { pooling decoder }\end{array}$ & Not defined & En, Fr, Es, De, Ru, It & $92.50 \%$ & $\begin{array}{l}\text { Used self-attentive pooling layer for } \\
\text { language identification task. }\end{array}$ & {$[28]$} \\
\hline 2021 & CNN LSTM & MFCC & $\begin{array}{l}\text { Iba, Kab, Sun, Ind, Eus, Jav, Tam, } \\
\text { Tel, Kan, Hin, Tha, Cnh, Eng, Por, } \\
\text { Mar, Rus }\end{array}$ & $74 \%$ & $\begin{array}{l}\text { CNN-LSTM combination is used for } \\
\text { predicting language. }\end{array}$ & {$[29]$} \\
\hline 2020 & $\begin{array}{l}\text { TDNN optimal } \\
\text { transport }\end{array}$ & MFCC & $\begin{array}{l}\text { Russian, Kazakh, Mandarin, } \\
\text { Korean, Japanese, Cantonese, } \\
\text { Vietnamese, Tibetan, Indonesian, } \\
\text { Uyghur }\end{array}$ & $\begin{array}{c}\text { Not } \\
\text { defined }\end{array}$ & $\begin{array}{l}\text { Used unsupervised technique joint } \\
\text { distribution adaptation neural network } \\
\text { model for spoken language identification. }\end{array}$ & {$[17]$} \\
\hline 2020 & $\begin{array}{l}\text { CRNN ResNet50 } \\
\text { DenseNet121 }\end{array}$ & Log-Mel & $\begin{array}{l}\text { Three different datasets with } \\
\text { different languages }\end{array}$ & $89 \%$ & $\begin{array}{l}\text { Used different pretrained models with } \\
\text { triplet entropy loss for improving the } \\
\text { generalization. }\end{array}$ & {$[16]$} \\
\hline 2020 & $\mathrm{CNN}$ & Log-Mel & $\begin{array}{l}\text { Slovene, Russian, Slovak, } \\
\text { Belarusian, Macedonian, Ukrainian, } \\
\text { Croatian, Bulgarian, Czech, Serbian, } \\
\text { Polish }\end{array}$ & $97.35 \%$ & $\begin{array}{l}\text { With the CNN model, two neural models } \\
\text { are made: baseline and robust models for } \\
\text { spoken language identification. }\end{array}$ & {$[18]$} \\
\hline 2020 & CapsNet & Log-Mel & $\begin{array}{l}\text { Arabic, Bengali, Chinese Mandarin, } \\
\text { English, Hindi, Turkish, Spanish, } \\
\text { Japanese, Punjabi, Portuguese }\end{array}$ & $98.20 \%$ & $\begin{array}{c}\text { Capsule network with encoder and } \\
\text { decoder works well on spoken language } \\
\text { identification. }\end{array}$ & {$[20]$} \\
\hline 2020 & CNN-LSTM & Log-Mel & Gujarati, Tamil, Telugu & $79.02 \%$ & $\begin{array}{l}\text { Used CNN-LSTM system that uses CTC } \\
\text { loss function at output layer and this } \\
\text { approach is used for spoken language } \\
\text { identification. }\end{array}$ & [19] \\
\hline 2020 & $\begin{array}{l}\text { Context aware } \\
\text { model }\end{array}$ & Log-Mel & $\begin{array}{c}\text { Prs, Amh, Fas, Hat, Hau, Eng, Cmn, } \\
\text { Fra, Rus, Hin, Ukr, Spa, Pus, Urd, } \\
\text { Yue, Bos, Vie, Hrv, Tur, Kat, Por, } \\
\text { Kor }\end{array}$ & $97 \%$ & $\begin{array}{l}\text { Context-aware model works well on pair } \\
\text { language and gives good results and } \\
\text { better accuracy. }\end{array}$ & {$[25]$} \\
\hline 2019 & ConvNets & MFCC & Fr, It, En, Ru, Es, De & $95.40 \%$ & $\begin{array}{l}\text { 2D ConvNets with attention and GRU } \\
\text { approach gives good results and better } \\
\text { accuracy. }\end{array}$ & {$[14]$} \\
\hline 2019 & ResNet50 & MFCC & Fr, It, En, Ru, Es, De & $89 \%$ & $\begin{array}{l}\text { In this, used pretrained ResNet50 model } \\
\text { and cyclic learning rate approach for } \\
\text { language identification. }\end{array}$ & {$[8]$} \\
\hline 2018 & $\begin{array}{l}\text { SVM-HMM } \\
\text { model }\end{array}$ & Not defined & Es, Fr, En, De & $70 \%$ & $\begin{array}{l}\text { In this, HMMs approach was used to } \\
\text { translate speech into the vector sequences } \\
\text { following the deep neural network. }\end{array}$ & {$[30]$} \\
\hline 2017 & $\begin{array}{l}\text { Inceptionv3 } \\
\text { CRNN }\end{array}$ & MFCC & Es, De, En, Fr & $96 \%$ & $\begin{array}{c}\text { The pipeline of inception-v3 based } \\
\text { transfer learning and bi-LSTM was used } \\
\text { to extract temporal and convolutional } \\
\text { attributes. }\end{array}$ & {$[24]$} \\
\hline 2010 & $\begin{array}{l}\text { Gaussian } \\
\text { mixture model }\end{array}$ & $\begin{array}{l}\text { Perceptual } \\
\quad \text { linear } \\
\text { prediction }\end{array}$ & $\begin{array}{l}\text { Tel, Dut, Hi, En, Ben, Fr, Es, De, Ru, } \\
\text { It }\end{array}$ & $88.80 \%$ & $\begin{array}{l}\text { The use of Gaussian mixture models with } \\
\text { the RPLP approach, which are processed } \\
\text { using PLP and MFCC features. }\end{array}$ & [3] \\
\hline 2009 & CNN-TDNN & MFCC & Fr, De, En & $91.20 \%$ & $\begin{array}{l}\text { Log-Mel images were used as features for } \\
\text { language identification coupled with SGD } \\
\text { based neural network. }\end{array}$ & [2] \\
\hline
\end{tabular}

common voice Kaggle [32], and Mozilla common voice dataset [33], which are described in Table 5.

Spoken language identification [30] dataset consists of 73080 train samples and 540 test samples with three languages: English, German, and Spanish. It contains both male and female recordings. The audio files have Free Lossless Audio Codec (FLAC) extensions. Language identification
[31] dataset consists of 22000 train samples with 22 languages: Arabic, Chinese, Dutch, English, Estonian, French, Hindi, Indonesian, Japanese, Korean, Latin, Persian, Portuguese, Pushto, Romanian, Russian, Spanish, Swedish, Tamil, Thai, Turkish, and Urdu. All data is stored in a CSV file with 1000 rows per language. Common voice Kaggle [32] dataset consists of 354785 audio samples, which are further 
TABLe 2: Architecture of 2D ConvNet model.

\begin{tabular}{|c|c|c|c|c|}
\hline Layers & Filters, kernels, and stride & Padding & Output & No. of parameters \\
\hline \multicolumn{5}{|l|}{ First block } \\
\hline Conv2D & $(32,7,7)$ & Valid & (None, 994, 34, 32) & 1600 \\
\hline BatchNorm & & & (None, $994,34,32$ ) & 128 \\
\hline MaxPool2D & $(3,3) s=2$ & Same & (None, $497,17,32$ ) & 0 \\
\hline \multicolumn{5}{|l|}{ Second block } \\
\hline Conv2D & $(64,5,5)$ & Same & (None, $497,17,64$ ) & 51264 \\
\hline BatchNorm & & & (None, $497,17,64)$ & 256 \\
\hline MaxPool2D & $(3,3) s=2$ & Same & (None, $249,9,64)$ & 0 \\
\hline \multicolumn{5}{|l|}{ Third block } \\
\hline Conv2D & $(128,3,3)$ & Same & (None, $249,9,128)$ & 73856 \\
\hline BatchNorm & & & (None, $249,9,128$ ) & 512 \\
\hline MaxPool2D & $(3,3) s=2$ & Same & (None, $125,5,128)$ & 0 \\
\hline \multicolumn{5}{|l|}{ Fourth block } \\
\hline Conv2D & $(256,3,3)$ & Same & (None, $125,5,256)$ & 295168 \\
\hline BatchNorm & & & (None, $125,5,256)$ & 1024 \\
\hline MaxPool2D & $(3,3) s=2$ & Same & (None, 63, 3, 256) & 0 \\
\hline \multicolumn{5}{|l|}{ Fifth block } \\
\hline Conv2D & $(512,3,3)$ & Same & (None, 63, 3, 512) & 1180160 \\
\hline BatchNorm & & & (None, $63,3,512$ ) & 2048 \\
\hline MaxPool2D & $(3,3) s=2$ & Same & (None, $32,2,512$ ) & 0 \\
\hline Flatten layer & & & (None, 32768) & 0 \\
\hline BatchNorm & & & (None, 32768) & 131072 \\
\hline Dense layer & 256 & & (None, 256) & 8388864 \\
\hline BatchNorm & & & (None, 256) & 1024 \\
\hline Dropout & 0.5 & & (None, 256) & 0 \\
\hline Dense layer & 3 & & (None, 3) & 771 \\
\hline
\end{tabular}

TABLE 3: Architecture of word embedding model.

\begin{tabular}{lcr}
\hline Layers & Output & No. of parameters \\
\hline Embedding layer & (None, 100,500) & 136984000 \\
Flatten & (None, 50000) & 0 \\
Dense layer & (None, 22) & 1100022 \\
\hline
\end{tabular}

TABLE 4: Evaluation metrics using the Bernoulli Naïve Bayes model.

\begin{tabular}{lr}
\hline Evaluation metrics & Score \\
\hline Precision & 0.946460 \\
Recall & 0.931159 \\
F1 score & 0.931159 \\
Matthews correlation coefficient & 0.925470 \\
\hline
\end{tabular}

TABle 5: Dataset description.

\begin{tabular}{|c|c|c|c|c|}
\hline Dataset & Spoken language identification [30] & $\begin{array}{c}\text { Language identification } \\
\text { dataset [31] }\end{array}$ & $\begin{array}{c}\text { Common voice Kaggle } \\
\text { dataset [32] }\end{array}$ & $\begin{array}{l}\text { Mozilla common } \\
\text { voice dataset [33] }\end{array}$ \\
\hline $\begin{array}{l}\text { Number of } \\
\text { languages }\end{array}$ & 3 & 22 & 16 & 4 \\
\hline Total samples & $\begin{aligned} \text { Train }= & 73080(420 \text { mins }) \text { Test }=540 \\
& (90 \text { mins })\end{aligned}$ & 22000 & 354785 & 23842 \\
\hline Type & Audio & Text & Audio & Audio and TSV \\
\hline Length & 10 seconds & 7 to 10 sentences in each line & Less than 10 seconds & $\begin{array}{l}\text { Less than } 10 \\
\text { seconds }\end{array}$ \\
\hline Extension & FLAC & CSV & Mp3 & Mp3 and TSV \\
\hline
\end{tabular}


divided into six folders with 16 main languages: United States English, Australian English, England English, Canadian English, Filipino, Hong Kong English, India, and South Asia, Irish English, Malaysian English, New Zealand English, Scottish English, Singaporean English, South Atlantic, South African, Welsh English, West Indies, and Bermuda; all languages are stored as $\mathrm{mp} 3$ files. It contains both male and female recordings. Mozilla common voice [33] dataset contains four languages, Estonian, Tamil, Turkish, and Mandarin, to check the robustness of the model. It includes 23842 audio samples, which are further divided into train samples and test samples. It contains both male and female recordings. This dataset is available in both audio clips and TSV files.

5.2. Hyperparameter Details. The attributes of the proposed method are represented in Table 6. The trial and error method is used while running the convolution neural network [8, 14], word embedding Keras [34, 35], and Naïve Bayes [36-38]. The selection of hyperparameter is also defined as an NP-complete problem [39, 40]. The efficient selection of hyperparameters can achieve better results $[41,42]$. In CNN, the epochs are set to be 60 , and the size of the batch is 32 with ReLU as an activation function. Dropouts $[43,44]$ are used with Adam optimizer. At the output layer, the softmax function [45] is used. In word embedding, it is a pretrained model by Keras. It is used in which the epochs are 25, and categorical cross-entropy loss is applied with Adam optimizer. Bernoulli naïve Bayes classifier is implemented with kernel function Bernoulli.

5.3. Performance Evaluation Metrics. The evaluation metrics are used for experimentation to check the performance of the model. Those are precision, recall, F1 score, and accuracy as shown in Table 7.

A receiver operating characteristic (ROC) curve is a graph that represents the classification model at different classification threshold values. These curves plot two parameters or attributes of ROC: false positive rate (FPR) and true positive rate (TPR).

In Figure 5, a multiclass ROC curve for language identification is presented. This spoken language identification Kaggle dataset contains three languages: German, English, and Spanish. Similarly, the ROC curve is also made like this for the language identification Kaggle dataset, which contains 22 languages: English, Arabic, French, Hindi, Urdu, Portuguese, Persian, Pushto, Spanish, Korean, Tamil, Turkish, Estonian, Russian, Romanian, Chinese, Swedish, Latin, German, Dutch, Japanese, and Thai.

\section{Results and Discussion}

The presented work discusses various methods which attain state-of-the-art results using four different datasets with audio, and the first dataset contains three languages, the second dataset includes 22 languages, and the third dataset includes 16 languages. All are available on the Kaggle and
TABLe 6: Parameter setting.

\begin{tabular}{lc}
\hline Proposed technique/parameters & Value \\
\hline Convolutional neural network & \\
Number of epochs & 60 \\
Batch size & 32 \\
Activation & ReLU \\
Optimizer & Adam \\
Dropouts & Yes \\
Loss & Categorical cross-entropy \\
Activation output & Softmax \\
Word embedding & \\
Number of epochs & 25 \\
CNN model & Word embedding Keras \\
Loss & Categorical cross-entropy \\
Optimizer & Adam \\
Activation output & Softmax \\
Naïve Bayes & \\
Type & Bernoulli Nä̈e Bayes \\
Kernel function & Bernoulli \\
\hline
\end{tabular}

fourth Mozilla common voice dataset contains four languages and is available on the Mozilla website. In the image domain, 2D convolutional neural networks obtained an accuracy of $98 \%$. In another dataset of CSV file, word embedding using the pretrained model obtained an accuracy of $95 \%$. With Bernoulli Naïve Bayes approach, we obtained an accuracy of $93 \%$ on a 22 -language dataset. Using the SVM and random forest classifier model achieved $82.88 \%$ and $72.42 \%$ accuracy on the 16-language dataset.

6.1. Misclassification. Various languages in the world belong to the Indo Persian and European families. In this group, the languages are separated into three subparts: Germanic, Romance, and Slavic. Our model confuses those languages with the same words; for example, "Cat" word in English, "Chatte" word in French, "Kat" word in Dutch, and "Katze" in German all have the same sound and pronunciation; hence, it is very difficult for a model to understand. Our model confuses Russian $(\mathrm{Ru})$ and French $(\mathrm{Fr})$ because they have similar accents; many words are adopted from French to Russian, so it is very difficult to give accurate results.

\subsection{Performance of Classification Model: Confusion Matrix.} In this section, the performance of the model is shown in Figure 6, using a confusion matrix for multiclass classification representing three classes of English, Spanish, and German. In this matrix, diagonal elements are predicted the same as the true value while nondiagonal elements are not classified properly by the model. On the $x$-axis, there is the true label, and on the $y$-axis, there is a predicted label.

In Figure 6, the multiclass confusion matrix for language identification in this spoken language identification Kaggle dataset is used, which contains three languages: German, English, and Spanish. Similarly, the confusion matrix is also made like this for the language identification Kaggle dataset, which includes 22 languages: English, Arabic, French, Hindi, Urdu, Portuguese, Persian, Pushto, 
TABLE 7: Different evaluation metrics.

\begin{tabular}{|c|c|c|c|c|}
\hline $\begin{array}{l}\text { Sr. } \\
\text { no. }\end{array}$ & Metrics & Equation & Remarks & Ref. \\
\hline 1 & $\begin{array}{l}\text { Accuracy } \\
(\mathrm{acc})\end{array}$ & $(t p+t n / t p+f p+t n+f n)$ & It is the ratio of correct outputs compared to the total number of outputs. & [30] \\
\hline 2 & Precision (p) & $(t p / t p+f p)$ & $\begin{array}{l}\text { It is the ratio of correct positive predictions from the total prediction from the } \\
\text { positive class. }\end{array}$ & {$[30]$} \\
\hline 3 & Recall (r) & $(t p / t p+t n)$ & $\begin{array}{c}\text { The recall is used to measure the fraction of positive patterns that are correctly } \\
\text { classified. }\end{array}$ & [30] \\
\hline 4 & F1 score $(\mathrm{FM})$ & $\left(2^{*} p^{*} r / p+r\right)$ & $\begin{array}{l}\text { The F1 score refers to or represents the harmonic mean between recall and } \\
\text { precision values. }\end{array}$ & {$[30]$} \\
\hline
\end{tabular}

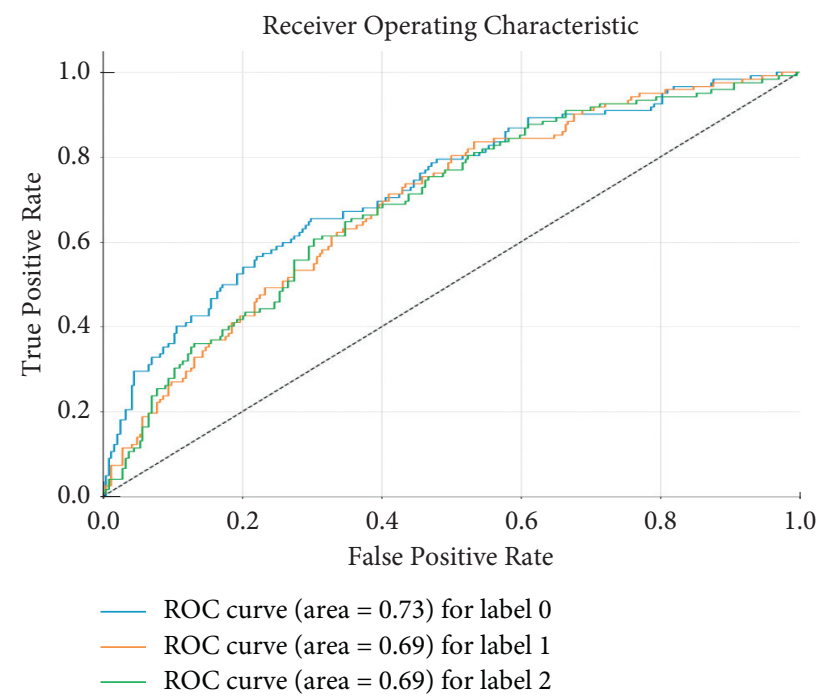

Figure 5: ROC curve.

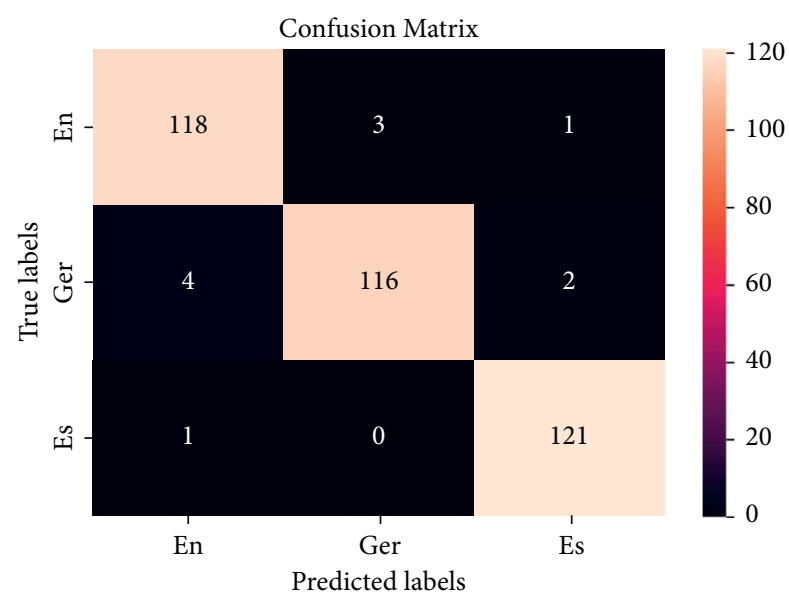

FIGURE 6: Confusion matrix for the multiclass spoken language identification.

Spanish, Korean, Tamil, Turkish, Estonian, Russian, Romanian, Chinese, Swedish, Latin, German, Dutch, Japanese, and Thai.
6.3. Performance Evaluation. Table 8 represents the performance comparison of various spoken language identification techniques using $\mathrm{CNN}$, Naïve Bayes, word 
TABLE 8: Performance measures obtained from various language identification techniques.

\begin{tabular}{|c|c|c|c|c|c|c|c|c|c|}
\hline Models & Dataset used & $\begin{array}{l}\text { Train } \\
\text { acc. }\end{array}$ & $\begin{array}{l}\text { Test } \\
\text { acc. }\end{array}$ & $\begin{array}{l}\text { Validation } \\
\text { acc. }\end{array}$ & Sensitivity & Specificity & $\begin{array}{c}\text { F1 } \\
\text { score }\end{array}$ & Precision & Recall \\
\hline $\mathrm{CNN}$ & $\begin{array}{l}\text { Spoken language } \\
\text { identification }\end{array}$ & 100 & 98.63 & 98.90 & 98.90 & 99 & 99.90 & 99.90 & 99.90 \\
\hline Naïve Bayes & $\begin{array}{c}\text { Language identification } \\
\text { dataset }\end{array}$ & 94 & 94 & 93 & 100 & 99 & 93.60 & 94.90 & 93.60 \\
\hline Word embedding & $\begin{array}{c}\text { Language identification } \\
\text { dataset }\end{array}$ & 95.50 & 92.20 & 93.20 & 98 & 99 & 92.50 & 93.50 & 92.50 \\
\hline Logistic regression & $\begin{array}{c}\text { Spoken language } \\
\text { identification }\end{array}$ & 64.32 & 61.95 & 63.55 & 64.23 & 63.55 & 64.23 & 64.23 & 64.23 \\
\hline Naïve Bayes & $\begin{array}{l}\text { Spoken language } \\
\text { identification }\end{array}$ & 50.25 & 49.75 & 51.20 & 52.32 & 51.78 & 51.20 & 51.20 & 51.20 \\
\hline SVM & Common voice Kaggle & 82.88 & 83.32 & 82.65 & 75.95 & 75.55 & 76.95 & 76.95 & 76.95 \\
\hline Random forest classifier & Common voice Kaggle & 72.42 & 71.90 & 71.50 & 66.32 & 67.24 & 67.23 & 67.23 & 67.23 \\
\hline VGG16 & Mozilla common voice & 81.30 & 80.21 & 81.05 & 81.25 & 80.52 & 79.83 & 80.02 & 80.02 \\
\hline ResNet50 & $\begin{array}{l}\text { Spoken language } \\
\text { identification }\end{array}$ & 86.30 & 80.20 & 84.32 & 85.36 & 84.23 & 84.65 & 82.75 & 82.75 \\
\hline CapsNet [20] & $\begin{array}{l}\text { Spoken language } \\
\text { identification }\end{array}$ & 91.80 & 88.76 & 90.72 & 88 & 89 & 89 & 89 & 89 \\
\hline $\begin{array}{l}\text { 2D ConvNet bidirectional } \\
\text { GRU [14] }\end{array}$ & $\begin{array}{l}\text { Spoken language } \\
\text { identification }\end{array}$ & 68.85 & 65.23 & 67.82 & 68 & 66 & 66 & 66 & 66 \\
\hline Acoustic model [25] & $\begin{array}{l}\text { Spoken language } \\
\text { identification }\end{array}$ & 75.69 & 73.23 & 74.23 & 75 & 75 & 75 & 75 & 75 \\
\hline CNN LSTM [19] & $\begin{array}{l}\text { Spoken language } \\
\text { identification }\end{array}$ & 83.25 & 80.52 & 82.45 & 83 & 82 & 82 & 82 & 82 \\
\hline $\begin{array}{l}\text { Logistic regression I- } \\
\text { vector [26] }\end{array}$ & Mozilla common voice & 84.30 & 80.23 & 82.52 & 78.95 & 80.05 & 82.36 & 82.36 & 82.36 \\
\hline LSTM-CNN [29] & Common voice Kaggle & 70.21 & 68.33 & 69.96 & 67.23 & 68.33 & 69.54 & 69.54 & 69.54 \\
\hline
\end{tabular}

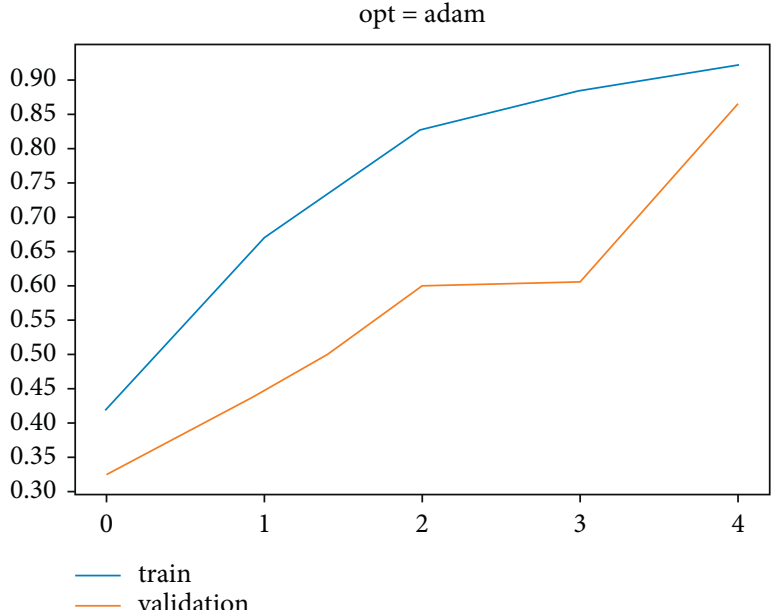

(a)

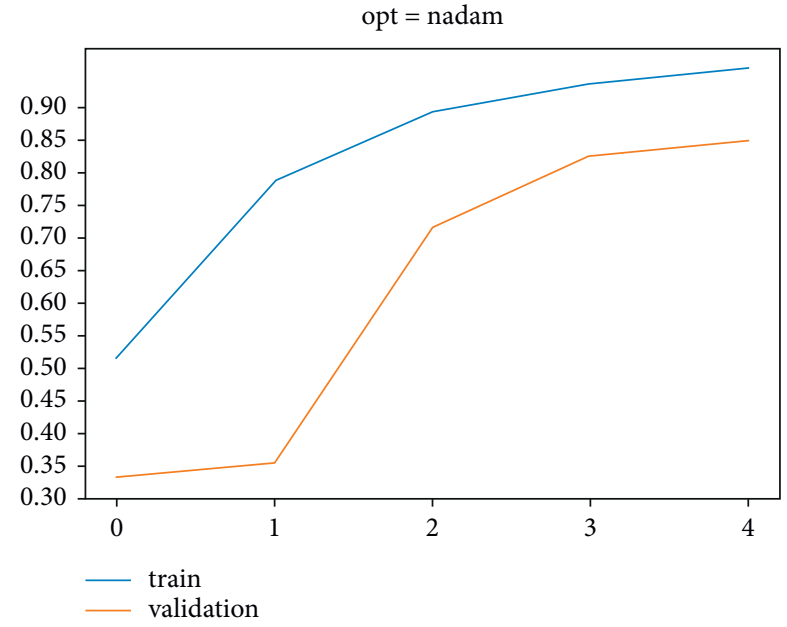

(b)

Figure 7: Continued. 


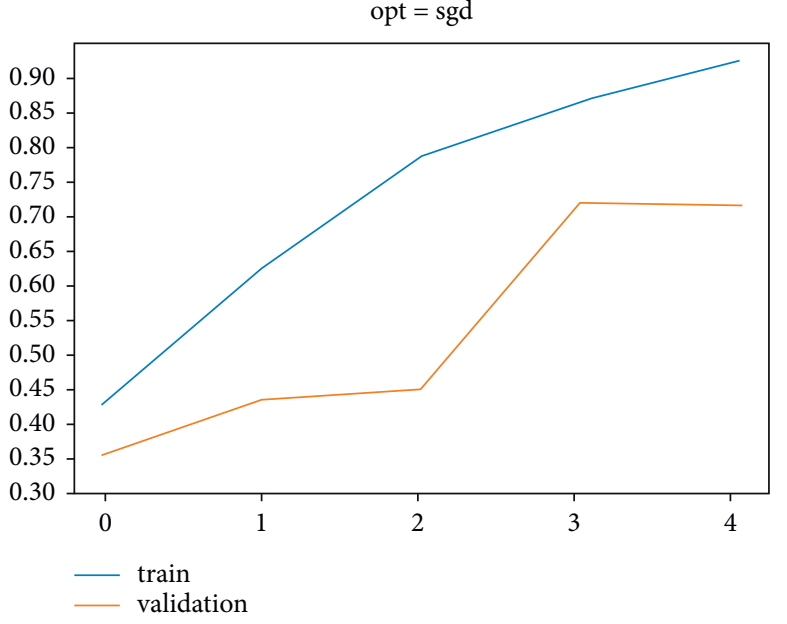

(c)

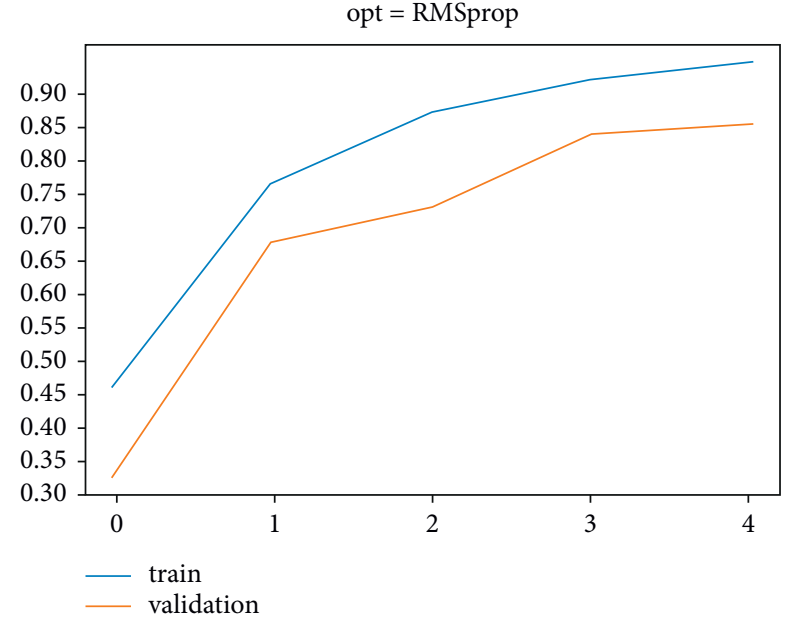

(d)

Figure 7: Use of different optimizers for comparison.

embedding, SVM, logistic regression, VGG16, ResNet50, and, random forest classifier. Spoken language identification contains three languages with train and test folders which include audio clips of different languages. Using CNN, the accuracy obtained $100 \%$ with good results and better achieved better precision, recall, and F1 score. The language identification dataset contains 22 languages in a CSV file which contains multiple sentences of each language. By using Naïve Bayes and word embedding techniques, the accuracy obtained $94 \%$ and $95 \%$ but is less efficient than the CNN technique. The Common voice Kaggle dataset contains 16 languages using SVM and random forest classifier techniques. The accuracy achieved is $82.88 \%$ and $72.42 \%$, which is good enough. The Mozilla common voice dataset contains four languages using VGG16 and logistic regression techniques. The accuracy obtained was $81.30 \%$ and 84.30 with good results.

6.4. Convergence for Training and Validation. This section is basically on the use of various optimizers on train and validation accuracy to compare our model. In Figure 7(a), the RMSprop optimizer with five epochs gives good results. In Figure 7(b), the use of Nadam optimizer with five epochs and its performance is not so good as compared to other optimizers. In Figure 7(c), the use of an SGD optimizer with five epochs and performance is a little bit better than the Nadam optimizer. In Figure 7(d), the use of Adam optimizer with five epochs also works well and gives good results.

\section{Conclusion and Future Scope}

There are two contributions of the paper in the field of spoken language identification. Firstly, we use the deep learning architecture for image classification in identifying languages from generated images from audio. Powerful performance can be achieved using relatively short files with minimum preprocessing. We believe that this model can be extended to more languages as long as sufficient. This approach achieved an accuracy of $98 \%$ and gave us good results. Secondly, we use the Bernoulli Naïve Bayes approach on a language identification dataset with 22 languages. It takes a little bit more time as compared to $\mathrm{CNN}$ in model fitting data. This approach gives us an accuracy of $93 \%$. And further, we apply another approach to this dataset, a pretrained model by Keras that is word embedding. It is a little bit faster and more accurate than Naïve Bayes. This approach achieved an accuracy of $95 \%$.

The performance of log-Mel spectrograms can be additionally refined by removing the noise from audio. There is a possibility for improvement by data augmentation on the available data using different methods like pitch shifting, crop, rotate, flip, adding random noise, and changing audio speed, and various methods. These help in making neural networks more robust to modifications that might be present in real-world scenarios. There is often further observation or review of various feature extraction techniques like Constant-Q transform and Fast Fourier Transform and their impact on language identification. These are known to possess a positive impact on the performance of convolutional neural networks.

\section{Data Availability}

The data that support the findings of this study are available upon request.

\section{Conflicts of Interest}

The authors declare that they have no conflicts of interest.

\section{Acknowledgments}

The authors would like to thank for the support of Taif University Researchers Supporting Project (no. TURSP2020/239), Taif University, Taif, Saudi Arabia. 


\section{References}

[1] E. Barnard and R. A. Cole, "Reviewing automatic language identification," IEEE Signal Processing Magazine, vol. 11, no. 4, pp. 33-41, 1994.

[2] G. Montavon, "Deep learning for spoken language identification," in Proceedings of the NIPS Workshop on Deep Learning for Speech Recognition and Related Applications, pp. 1-4, Vancouver, Canada, December 2009.

[3] P. Kumar, A. Biswas, A. N. Mishra, and M. Chandra, "Spoken language identification using hybrid feature extraction methods," vol. 1, no. 2, pp. 11-15, 2010, https://arxiv.org/abs/ 1003.5623 .

[4] B. M. L. Srivastava, H. K. Vydana, A. K. Vuppala, and M. Shrivastava, "A language model based approach towards large scale and lightweight language identification systems," pp. 4-7, 2015, https://arxiv.org/abs/1510.03602.

[5] P. Shen, X. Lu, S. Li, and H. Kawai, "Conditional generative adversarial nets classifier for spoken language identification," in Proceedings of the Annual Conference of the International Speech Communication Association, pp. 2814-2818, INTERSPEECH, Stockholm, Sweden, August 2017.

[6] M. Zampieri, A. M. Ciobanu, and L. P. Dinu, "Native language identification on text and speech," 2017, https://arxiv.org/abs/ 1707.07182.

[7] W. M. Campbell, F. Richardson, and D. A. Reynolds, "Language recognition with word lattices and support vector machines," in Proceedings of the 2007 IEEE International Conference on Acoustics, Speech and Signal Processing-ICASSP '07, vol. 4, no. 2, pp. 989-992, Honolulu, HI, USA, April 2007.

[8] S. Revay, M. Teschke, and Novetta, "Multi-class language identification using deep learning on spectral images of audio signals," pp. 1-7, 2019, https://arxiv.org/abs/1905.04348.

[9] M. Masud, G. Muhammad, H. Alhumyani et al., "Deep learningbased intelligent face recognition in IoT-cloud environment," Computer Communications, vol. 152, pp. 215-222, 2020.

[10] S. Sharma and V. Kumar, "Low-level features based 2D face recognition using machine learning," International Journal of Intelligent Engineering Informatics, vol. 8, no. 4, pp. 305-330, 2020.

[11] S. Sharma and V. Kumar, "Transfer learning in $2.5 \mathrm{D}$ face image for occlusion presence and gender classification," in Handbook of Research on Deep Learning Innovations and Trends, pp. 97-113, IGI Global, Hershey, PA, USA, 2019.

[12] M. Kaur and D. Singh, "Multi-modality medical image fusion technique using multi-objective differential evolution based deep neural networks," Journal of Ambient Intelligence and Humanized Computing, vol. 12, no. 2, pp. 2483-2493, 2021.

[13] M. Kaur and D. Singh, "Multiobjective evolutionary optimization techniques based hyperchaotic map and their applications in image encryption," Multidimensional Systems and Signal Processing, vol. 32, no. 1, pp. 281-301, 2021.

[14] S. S. Sarthak, S. Shukla, and G. Mittal, "Spoken language identification using convNets," Lecture Notes in Computer Science (including subseries Lecture Notes in Artificial Intelligence and Lecture Notes in Bioinformatics), vol. 11912, pp. 252-265, 2019, LNCS.

[15] I. Lopez-moreno, J. Gonzalez-dominguez, O. Plchot, D. Martinez, J. Gonzalez-rodriguez, and P. Moreno, "Google inc., New York, USA ATVS-biometric recognition group, universidad autonoma de madrid, Spain brno university of technology, czech republic aragon institute for engineering research ( I3A ), university of Zaragoza, Spain,” pp. 0-4, 2014.
[16] R. van der Merwe, "Triplet entropy loss: improving the generalisation of short speech language identification systems," 2020, https://arxiv.org/abs/2012.03775.

[17] X. Lu, P. Shen, Y. Tsao, and H. Kawai, "Unsupervised neural adaptation model based on optimal transport for spoken language identification," no. 19, , 2020, https://arxiv.org/abs/ 2012.13152 .

[18] B. M. Abdullah, J. Kudera, T. Avgustinova, B. Möbius, and D. Klakow, "Rediscovering the slavic continuum in representations emerging from neural models of spoken language identification," 2020, https://arxiv.org/abs/2010.11973.

[19] P. Rangan, S. Teki, and H. Misra, "Exploiting spectral augmentation for code-switched spoken language identification," no. 2, 2020, https://arxiv.org/abs/2010.07130.

[20] M. Verma and A. B. Buduru, "Fine-grained language identification with multilingual CapsNet model," in Proceedings of the-2020 IEEE 6th International Conference on Multimedia Big Data (BigMM), pp. 94-102, New Delhi, India, September 2020.

[21] M. Z. Ansari, T. Ahmad, and A. Fatima, "Feature selection on noisy twitter short text messages for language identification," no. 1, https://arxiv.org/abs/2007.05727, 2020.

[22] S. Punjabi, H. Arsikere, Z. Raeesy et al., "Streaming end-toend bilingual ASR systems with joint language identification," 2020, https://arxiv.org/abs/2007.03900.

[23] C. Chandak, Z. Raeesy, A. Rastrow et al., "Streaming language identification using combination of acoustic representations and ASR hypotheses," 2020, https://arxiv.org/abs/2006.00703.

[24] S. Wang, L. Wan, Y. Yu, and I. L. Moreno, "Signal combination for language identification," 2019, https://arxiv.org/ abs/1910.09687.

[25] A. Titus, J. Silovsky, N. Chen, R. Hsiao, M. Young, and A. Ghoshal, "Improving language identification for multilingual speakers," in Proceedings of the ICASSP 2020-2020 IEEE International Conference on Acoustics, Speech and Signal Processing (ICASSP), pp. 8284-8288, Barcelona, Spain, May 2020.

[26] A. I. Abdurrahman and A. Zahra, "Spoken language identification using $i$-vectors, $x$-vectors," Bulletin of Electrical Engineering and Informatics, vol. 10, no. 4, pp. 2237-2244, 2021.

[27] E. Salesky, B. M. Abdullah, S. Mielke et al., "SIGTYP 2021 shared task: robust spoken language identification," in Proceedings of the Third Workshop on Computational Typology and Multilingual NLP, Association for Computational Linguistics, pp. 122-129, Mexico city, Mexico, 2021.

[28] R. Bedyakin, "Low-resource spoken language identification using self-attentive pooling and deep 1D time-channel separable convolutions," 2021, http://arxiv.org/abs/2106. $00052 \mathrm{v} 1$.

[29] A. Scherbakov, L. Whittle, R. Kumar, S. Singh, M. Coleman, and E. Vylomova, "Anlirika: an LSTM-CNN flow twister for spoken language identification," in Proceedings of the Third Workshop on Computational Typology and Multilingual NLP, Association for Computational Linguistics, pp. 145-148, Mexico city, Mexico, 2021.

[30] Spoken Language Identification | Kaggle, 2021, https://www. kaggle.com/toponowicz/spoken-language-identification.

[31] Language Identification dataset | Kaggle, 2021, https://www. kaggle.com/zarajamshaid/language-identification-datasst.

[32] Common Voice | Kaggle, 2021, https://www.kaggle.com/ mozillaorg/common-voice.

[33] Common Voice, 2021, https://commonvoice.mozilla.org/en.

[34] T. Ranasinghe and M. Zampieri, "Multilingual offensive language identification with cross-lingual embeddings," 2020, https://arxiv.org/abs/2010.05324. 
[35] C. Orasan, "Aggressive language identification using word embeddings and sentiment features," in Proceedings of the First Workshop on Trolling, Aggression and Cyberbullying (\{TRAC\}-2018), pp. 113-119, August 2018, https://www. aclweb.org/anthology/W18-4414.

[36] T. Gottron and N. Lipka, "A comparison of language identification approaches on short, query-style texts," Lecture Notes in Computer Science (Including Subseries Lecture Notes in Artificial Intelligence and Lecture Notes in Bioinformatics), vol. 5993, pp. 611-614, 2010, LNCS.

[37] T. Baldwin and M. Lui, "Language identification: the long and the short of the matter," in Proceedings of the NAACL HLT 2010-Human Language Technologies: the 2010 Annual Conference of the North American Chapter of the Association for Computational Linguistics, Proceedings of the Main Conference, pp. 229-237, Los Angeles, CA, USA, June 2010.

[38] M. Lui and T. Baldwin, "Langid.py: an off-the-shelf Language identification tool," Aclweb.Org, pp. 25-30, 2012, http://www. aclweb.org/anthology-new/P/P12/P12-3005.pdf.

[39] H. Kaushik, D. Singh, M. Kaur, H. Alshazly, A. Zaguia, and H. Hamam, "Diabetic retinopathy diagnosis from fundus images using stacked generalization of deep models," IEEE Access, vol. 9, pp. 108276-108292, 2021.

[40] Y. Xu and T. Qiu, "Human activity recognition and embedded application based on convolutional neural network," Journal of Artificial Intelligence and Technology, vol. 1, no. 1, pp. 51-60, 2021.

[41] D. Jiang, G. Hu, G. Qi, and N. Mazur, “A fully convolutional neural network-based regression approach for effective chemical composition analysis using near-infrared spectroscopy in cloud," Journal of Artificial Intelligence and Technology, vol. 1, no. 1, pp. 74-82, 2021.

[42] S. Ghosh, P. Shivakumara, P. Roy, U. Pal, and T. Lu, "Graphology based handwritten character analysis for human behaviour identification," CAAI Transactions on Intelligence Technology, vol. 5, no. 1, pp. 55-65, 2020.

[43] B. Gupta, M. Tiwari, and S. Singh Lamba, "Visibility improvement and mass segmentation of mammogram images using quantile separated histogram equalisation with local contrast enhancement," CAAI Transactions on Intelligence Technology, vol. 4, no. 2, pp. 73-79, 2019.

[44] M. Masud, N. Sikder, A. A. Nahid, A. K. Bairagi, and M. A. AlZain, "A machine learning approach to diagnosing lung and colon cancer using a deep learning-based classification framework," Sensors, vol. 21, no. 3, p. 748, 2021.

[45] G. Hu, S. H. K. Chen, and N. Mazur, "Deep neural networkbased speaker-aware information logging for augmentative and alternative communication," Journal of Artificial Intelligence and Technology, vol. 1, no. 2, pp. 138-143, 2021. 